(C) 2018 EDIZIONI MINERVA MEDICA

Online version at http://www.minervamedica.it

Panminerva Medica 2018 mese;60(0):000-000

DOI: $10.23736 / \mathrm{S} 0031-0808.18 .03459-6$

\section{Targeting microRNAs using nanotechnology in pulmonary diseases}

MicroRNAs (miRNAs) are short non-coding RNAs, which control gene expression post-transcriptionally by directly blocking translation of their target messenger RNAs (mRNAs) or by repressing protein production via mRNA destabilization. In the present times, the research in the area of targeting miRNAs using Antagomirs, which are also known as anti-miRs as potential therapeutic moieties for the management of various chronic respiratory diseases, including asthma, chronic obstructive pulmonary disease (COPD), and cystic fibrosis (CF), is gaining enormous attention. The applications and concepts of novel drug delivery systems such as nanoparticles can be employed in administering identified biological moieties, including specific antagomirs, which can help in achieving the therapeutic effectiveness by providing the targeted effects and overcoming the various problems like biofilms etc associated with various respiratory diseases.

Majority of the existing studies with nanoparticles containing anti-miRs were employed in the area of cancer research and limited information is available in the context of pulmonary diseases. Various types of nanoparticles such titanium dioxide or surfacemodified gold nanoparticles can be employed as a carrier to deliver various antagomirs to target the specific miRNAs. Yung et $a l$. demonstrated the efficacy of lipid nanoparticles composed of quaternary amine-tertiary amine cationic lipid combination (QTsome) to deliver AntimiR-21, an oligonucleotide complementary to miR-21 in lung cancer. The prepared nanoparticles showed tumor regression, prolonged survival, and miR-21 target upregulation in an A549 xenograft mouse model. ${ }^{2}$ Another study has shown the importance of lipid nanoparticles as a carrier for the microRNA-145 inhibitor in repairing pulmonary arteriopathy and improving cardiac function in rats with severe pulmonary arterial hypertension (PAH). Overall, their results indicated to be effective and having low toxicity with the pulmonary delivery of miRNA-145 inhibitor using functionalized cationic lipopolyamine nanoparticles. ${ }^{3}$

Polyethyleneimine and chitosan tripolyphosphate nanoparticles encapsulating hsa-miR-126 and hsa-miR-145 demonstrated effective delivery of the miRNAs to CFBE41o- (human F508del cystic fibrosis transmembrane conductance regulator bronchial epithelial) cells to modulate the gene expression in treating cystic fibrosis. These nanoparticles proved effective and biocompatible. ${ }^{4}$

Various new advancements in the pulmonary drug delivery include triggered release lipid nanocapsules, sponges and biomolecular corona, which can be used to load various drugs. Various cell-derived membrane vesicles (CMVs) namely, microvesicles and exosomes also help in transporting various proteins and nucleic acids. Such recent developments may be employed to encapsulate various antagomirs in treating the pulmonary diseases. Recently, Dahlman et al. developed a method to measure the biodistribution of various nanoparticles by formulating the nanoparticles carrying specific nucleic acid barcodes that can be sequenced to understand and quantify the particle biodistribution. Their study also provides information on the chemical structure and in-vivo delivery of nanoparticles, which can help in designing the nanoparticles with highly targeted effects in specific tissues and cells. ${ }^{5}$

miRNA-based therapy is expanding its horizons for the treatment of pulmonary diseases where the nanotechnology is an advanced platform for delivering such therapeutic moieties including antagomirs to target various miRNAs involved in the respiratory disease pathology. Moreover, nanoparticles and similar vesicular drug delivery systems can also be used as carriers for small interfering RNAs (siRNAs). This novel drug delivery system offers great benefits such as targeted effects, lesser side and unwanted effects with maximal efficacy and improved patient compliance. Application of nanotechnology still warrants attention and in-depth investigations especially the class focussed on targeting miRNAs in various chronic inflammatory lung diseases, which can bring a new revolution to the pulmonary clinic.

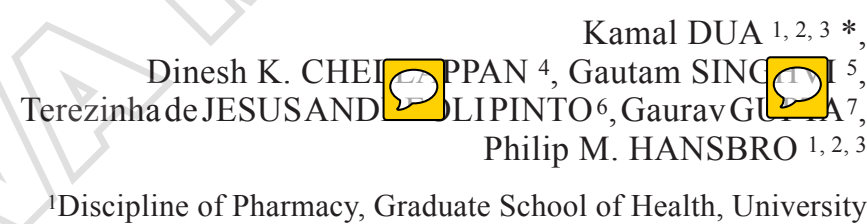
Discipline of Pharmacy, Graduate School of Health, University Sciences and Pharmacy, The University of Newcastle, Callaghan, Australia; ${ }^{3}$ The Priority Research Centre for Healthy Lungs, Hunter Medical Research Institute, Newcastle, Australia; ${ }^{4}$ Department of Life Sciences, School of Pharmacy, International Medical University, Bukit Jalil, Malaysia; ${ }^{5}$ Department of Pharmacy, Birla Institute of Technology and Science (BITS),

Pilani, India; ${ }^{6}$ Faculty of Pharmaceutical Sciences, University of São Paulo, São Paulo, Brazil; ${ }^{7}$ School of Pharmaceutical Sciences, Jaipur National University, Jagatpura, India

*Corresponding author: Kamal Dua, Discinling of Pharmacy, Graduate School of Health, University of Technolog Sy ney, Ultimo NSW 2007, Australia. E-mail: kamalpharmacist02@gma 2

\section{References}

1. Dua K, Shukla SD, Tekade RK, Hansbro PM. Whether a novel drug delivery system can overcome the problem of biofilms in respiratory diseases? Drug Deliv Transl Res 2017;7:179-87.

2. Yung BC, Li J, Zhang M, Cheng X, Li H, Yung EM, et al. Lipid 
Nanoparticles Composed of Quaternary Amine-Tertiary Amine Cationic Lipid Combination (QTsome) for Therapeutic Delivery of AntimiR-21 for Lung Cancer. Mol Pharm 2016;13:653-62.

3. McLendon JM, Joshi SR, Sparks J, Matar M, Fewell JG, Abe K, et al. Lipid nanoparticle delivery of a microRNA-145 inhibitor improves experimental pulmonary hypertension. J Control Release 2015;210:6775.

4. McKiernan PJ, Cunningham O, Greene CM, Cryan SA. Targeting miRNA-based medicines to cystic fibrosis airway epithelial cells using nanotechnology. Int J Nanomedicine 2013;8:3907-15.

5. Dahlman JE, Kauffman KJ, Xing Y, Shaw TE, Mir FF, Dlott CC, et al.
Barcoded nanoparticles for high throughput in vivo discovery of targeted therapeutics. Proc Natl Acad Sci USA 2017;114:2060-5.

Conflicts of interest.-The authors certify that there is no conflict of interest with any financial organization regarding the material discussed in the manuscript.

Manuscript accepted: May 4, 2018. - Manuscript received: April 17, 2018.

(Cite this article as: Dua K, Chellappan DK, Singhvi G, de Jesus Andreoli Pinto T, Gupta G, Hansbro PM. Targeting microRNAs using nanotechnology in pulmonary diseases. Panminerva Med 2018;60:000-000. DOI: 10.23736/ S0031-0808.18.03459-6)

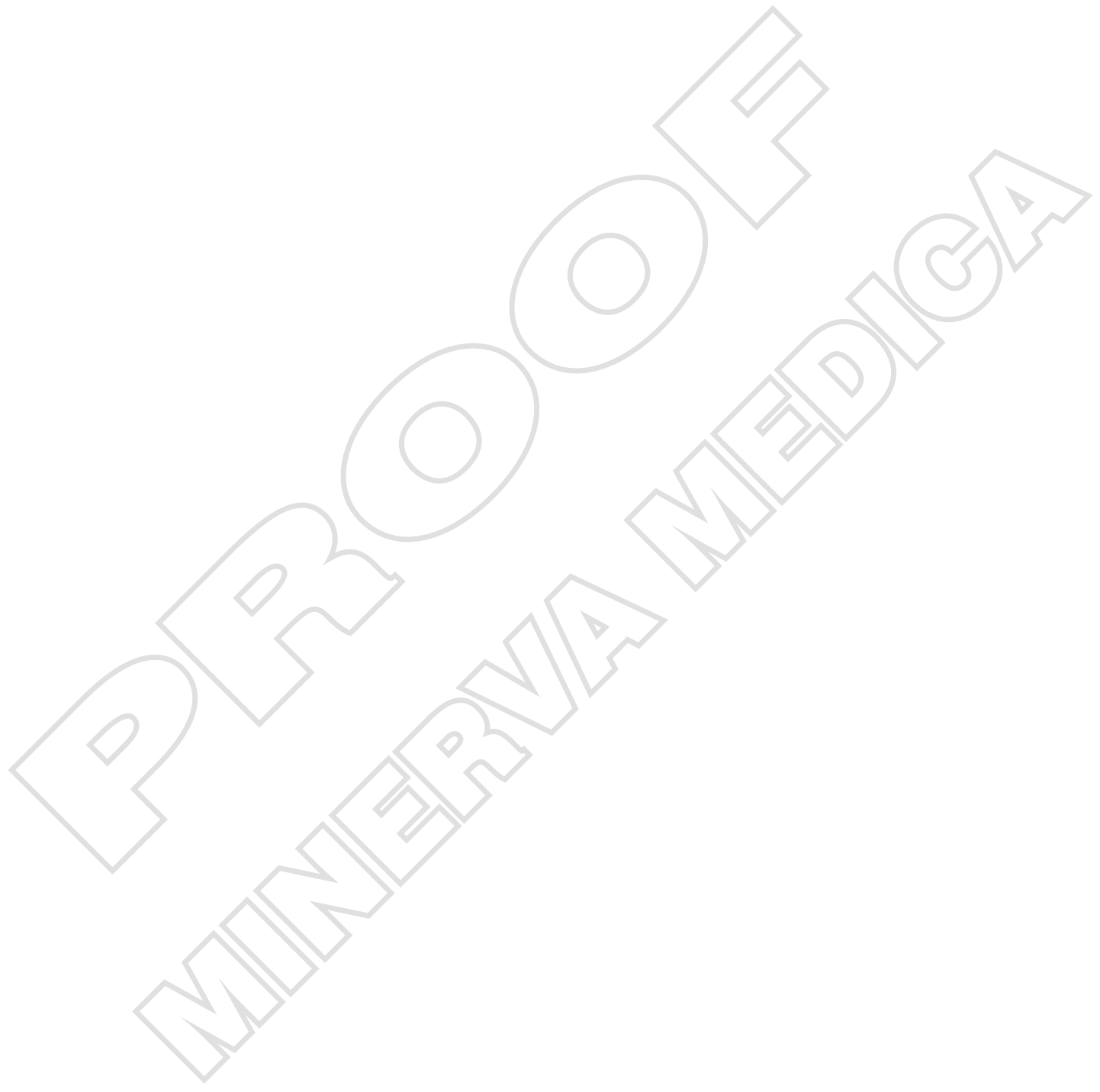

